\title{
Migración transnacional colombiana y estrategias políticas online. Reproducción de la comunidad a través de la vinculación transnacional*
}

\author{
Colombian Transnational Migration and Political Strategies \\ Online. Community Reproduction through Transnational \\ Linkages
}

\section{Migração Transnacional Colombiana e estratégias políticas online. Reprodução comunidade a través da vinculação transnacional} Yolanda González Rábago* • España

Recibido el 10 de agosto de 2011, aceptado el 6 de diciembre de 2011

\section{Resumen}

Objetivo: analizar la política migratoria colombiana en relación con las teorías de vinculación transnacional que se desarrollan en el ámbito de la sociología de las migraciones, partiendo de la idea de que las nuevas tecnologías proporcionan una infraestructura sobre las que los países de origen crean cauces de extensión y difusión de la política y la nación hacia los lugares de destino y, especialmente, en cada uno de sus emigrados. Metodología: se hace un análisis cuantitativo del número, tipo y composición de las redes y los usuarios que participan en la Plataforma web RedEsColombia, y un análisis cualitativo-discursivo de la información audiovisual que se puede recoger de la propia web. Resultados: RedEsColombia ha supuesto un éxito por el número de usuarios registrados, mientras resulta más cuestionable la incidencia de la vinculación que genera el Portal. Así mismo, quedan claros los ejes discursivos en torno a los que gira la política colombiana en materia de migraciones, teniendo como objetivo último la vinculación de los emigrados para su contribución al desarrollo del país. Conclusiones: la utilización de las TIC en el Estado colombiano pretende la difusión y la extensión de los programas de vinculación nacional de los emigrados, mediante la creación de un sentimiento de pertenencia y comunidad.

Palabras claves: Emigración, transnacional, Colombia, políticas, TIC.

\footnotetext{
* Este artículo es parte del trabajo realizado para la tesis en curso titulada "Migraciones transnacionales y redes sociales telemáticas. Incidencia sobre la integración social de los migrantes en las sociedades de destino”, que se ubica en el Departamento de Sociología 2 de la Universidad del País Vasco, bajo la dirección de Cristina Blanco F. de Valderrama. La autora disfruta de una Ayuda para la Formación y Perfeccionamiento de Personal Investigador financiada por el Departamento de Educación, Universidades e Investigación del Gobierno Vasco (BOPV, 08-01-2010).

** Licenciada en Sociología. Magíster en Migraciones e investigadora en la Universidad del País Vasco. Pertenece a Xenia, Grupo de Investigación en Migraciones, Alteridad y Desarrollo Humano, de la Universidad del País Vasco (España). Actualmente desarrolla su tesis doctoral en migraciones transnacionales y nuevas tecnologías de la información y la comunicación. Correo electrónico: yolanduca@yahoo.es
}

Para citar este artículo:
González, Y. (junio, 2012). Migración transnacional colombiana y estrategias políticas online. Reproducción de la comunidad a través de la vinculación transnacional. Ánfora, 19 (32), 69 - 90. Universidad Autónoma de Manizales. ISSN 0121-6538. 


\begin{abstract}
Objective: this research paper aims at analyzing the Colombian migration policy in relation to theories of transnational agreements developed in the field of sociology of migrations. This is based on the idea that new technologies provide an infrastructure on which the countries of origin create channels of outreach and dissemination of policy and government institutions to the destination places, especially, to each emigrant. Methodology: it applies a quantitative analysis of the number, type and composition of networks and users involved in the web platform RedEsColombia and discursive qualitative analysis of audiovisual information collected from this web. Results: re$\mathrm{dEsColombia}$ has been successful thanks to the number of registered users, whereas the impact of the relationship created by the Portal is more questionable. Likewise, the discursive axes around the Colombian policy on migration are clear, being its ultimate objective to have the emigrants contribute to the national development. Conclusions: the use of ICTs by the Colombian government seeks the dissemination and outreach of national outreach programs oriented to emigrants by creating a sense of belonging and community.
\end{abstract}

Keywords: Migration, transnational, Colombia, policies, ICT.

\title{
Resumo
}

Objetivo: analisar a política migratória em relação com as teorias de vinculação transnacional que se desenvolvem no âmbito da sociologia das migrações, partindo da idéia de que as novas tecnologias proporcionam uma infra-estrutura sobre as que os países de origem criam fontes de extensão e difusão da política e a nação para os lugares de destino e, especialmente, em cada um de seus emigrados. Metodologia: faz se um analise quantitativo do numero, tipo e composição das redes e aos usuários que participam na Plataforma web Redcolombiana e um analise qualitativo-discursivo da informação audiovisual que se recolhe da própria web. Resultados: RedEscolombia tem posto um sucesso pelo numero de usuários registrados, enquanto resulta mais questionável a incidência da vinculação que gera o Portal. Assim mesmo, ficam claros os eixos discursivos em torno aos que a política colombiana em matéria de migrações, tendo como objetivo ultima dos emigrados para sua contribuição ao desenvolvimento do país. Conclusões: a utilização das TIC no Estado colombiano pretende a difusão e a extensão dos programas de vinculação nacional dos emigrados, mediante a criação de um sentimento de pertencia e comunidade.

Palavras-Chaves: emigração, transnacional, Colombia, políticas, TIC.

González, Y. (junio, 2012). Migración transnacional colombiana y estrategias políticas online. Reproducción de la comunidad a través de la vinculación transnacional. Ánfora, 19 (32), 69 - 90. Universidad Autónoma de Manizales. ISSN 0121-6538. 


\section{Introducción}

Desde hace algún tiempo, los países de emigración, que en muchos casos son también de inmigración, han comenzando a conocer en mayor medida y a interesarse cada vez más acerca de cómo y dónde se encuentran sus nacionales en el extranjero, lo que siempre se ha denominado la diáspora ${ }^{1}$. Del mismo modo, empieza a darse una preocupación por el papel que estos juegan, tanto en el desarrollo de sus países como en la representación que ejercen en el exterior. Los gobiernos de los países de origen han empezado a ser conscientes del gran volumen de personas que han emigrado y de los múltiples motivos que les han llevado a hacerlo y, sobre todo, de las consecuencias que para dichos países puede tener este hecho.

En la actualidad, a pesar de que la visión positiva de la emigración no ha cambiado en términos generales y la política migratoria de salida no se ha modificado a grandes rasgos, los países de origen sí han dado un paso adelante en su perspectiva, y han empezado a ver las potencialidades que las comunidades de emigrantes tienen sobre el desarrollo del país de origen y se han sensibilizado sobre la situación que sus nacionales viven en las sociedades receptoras. Además, se percibe una preocupación gubernamental por la protección de los emigrantes que, en su génesis, ha sido siempre parte de las obligaciones que los Estados-nación tiene para con sus nacionales, pero que, en la realidad, no se ha cumplido en la mayoría de los casos (bien sea por déficit de medios o por falta de voluntad para hacerlo).

Con este nuevo enfoque de origen, los países están aprovechando las oportunidades que el nuevo sistema tecnológico TIC ofrece, de manera que han dado el salto a las nuevas tecnologías de la información y la comunicación, no sólo como artefactos que facilitan el contacto, sino también profundizando en la creación de un nuevo tipo de relación de interdependencia entre el Estado y su población, con especial relevancia de las personas residentes fuera del país.

La puesta en marcha de políticas migratorias, o más exactamente, de programas de intervención pública dedicados a la migración, en especial a las personas emigrantes que se encuentran en el exterior, es un ejemplo más de la consolidación de la perspectiva transnacional en el estudio de las migraciones internacionales de un actor fundamental en la dinámica como son los países de origen. Colombia representa el paradigma de

1 Existe cierto debate entre aquellos que le apuestan al término diáspora para seguir hablando de los grupos de emigrantes, mientras que otros prefieren llamarlos comunidades transnacionales. Si bien el primero tiene fuertes connotaciones míticas (judíos, gitanos, etc.) y se ha caracterizado por la dispersión en varios lugares, el segundo atiende más a grupos que tienden a seguir un patrón de concentración y con una fuerte vinculación nacional (Cortés y Sanmartín, 2008). Las políticas sobre migración que Estados como el colombiano están promoviendo, han elegido utilizar el término comunidad para referirse a sus nacionales en el exterior.

Para citar este artículo:
González, Y. (junio, 2012). Migración transnacional colombiana y estrategias políticas online. Reproducción de la comunidad a través de la vinculación transnacional. Ánfora, 19 (32), 69 - 90. Universidad Autónoma de Manizales. ISSN 0121-6538. 
dicho enfoque debido al ímpetu con que se ha lanzado a la temática y en concreto por la utilización que hace de las posibilidades que brindan las TIC.

La investigación de la que da cuenta este artículo tiene como objetivo fundamental poner de relieve estos procesos que tienen lugar en los países emisores de migración, en concreto en el caso colombiano, mediante el análisis del Programa Colombia Nos Une y la plataforma web RedEsColombia. En concreto, busca analizar la política migratoria colombiana en relación con las teorías de vinculación transnacional que vienen desarrollándose en el ámbito de la sociología de las migraciones, partiendo de la idea de que las nuevas tecnologías proporcionan una infraestructura sobre las que los países de origen crean cauces de extensión y difusión de la política y la nación hacia los lugares de destino $y$, especialmente, en cada uno de sus emigrados.

\section{Extensión de la (trans-) nación más allá de las fronteras}

En los últimos años, los países de origen han puesto en marcha lo que pretenden ser políticas integrales de migración que combinen la tradicional atención a los nacionales en el extranjero a través de embajadas y consulados, con una mayor política activa de sensibilización sobre el fenómeno migratorio en el propio país de origen, y una apertura hacia nuevos servicios para los ya emigrados, que atiendan demandas referidas a temas sanitarios, de pensiones, educativos, laborales, de promoción cultural, políticos, asociativos y de seguridad.

Así mismo, con esa intención de atender, apoyar, asistir y proteger a los compatriotas en el extranjero, se presenta también una posibilidad de conocer dónde y cómo se encuentran sus diásporas y saber cuál es su relación con el país de origen, para así ejercer cierto tipo de control sobre las mismas y aprovechar en la medida de lo posible su potencial de influencia y capital social a favor de los intereses del país emisor. Esta nueva conciencia sobre la importancia de proteger a los compatriotas residentes en terceros países se presenta como una buena oportunidad para fortalecer el sentimiento nacional, con respecto a los que todavía viven en el país y, sobre todo, entre aquellos que residen más allá de las fronteras nacionales.

Las estrategias de vinculación de los países de origen tiene como población diana a las comunidades (trans)nacionales residentes en el exterior, pero no sólo van dirigidas a incrementar la relación con éstas, sino que están fomentando, en la práctica, la mayor conexión entre el Estado y la población residente en el país no emigrada, creando un sentimiento de pertenencia, es decir, una sensibilidad que va más allá del hecho de haber abandonado el país y que ahonda en la creación de una nación por encima de las fronteras del Estado. De alguna manera, y al tomar el nombre de transnacional, estas estrategias no superan, sin embargo, la perspectiva del nacionalismo criticada por Ulrick Beck, esto es: "la continuidad, más allá del periodo histórico de su vigencia, de la asunción de la equiparación entre Estado, sociedad nacional y territorio” (Beck 2005, pp. 38-39).

Para citar este artículo:
González, Y. (junio, 2012). Migración transnacional colombiana y estrategias políticas online. Reproducción de la comunidad a través de la vinculación transnacional. Ánfora, 19 (32), 69 - 90. Universidad Autónoma de Manizales. ISSN 0121-6538. 
A pesar de que el lugar por el que transitan los migrantes es un espacio plurilocal o multiterritorial, un espacio imaginado (Anderson 1983), un lugar que sobrepasa las fronteras físicas, la perspectiva desde la que actúan, tanto los propios migrantes, como las instituciones del Estado, no deja de ser la de un nacionalismo metodológico que no supera el concepto de nación sino que lo extiende sin circunscribirse al territorio físico.

Como expresan Wimmer y Glick Schiller (2003), la tendencia del nacionalismo metodológico de aceptar al Estado-nación y a sus fronteras como la variable clave en el análisis social, sigue influyendo a los países emisores de migrantes que siguen pretendiendo la relación con sus emigrados bajo una lógica nacionalista, mientras, a la vez, la investigación centrada en cuestiones transnacionales tampoco ha dejado el prisma del nacionalismo para el abordaje de fenómenos que transcienden el Estado-nación. El denominado espacio transnacional supera la nación pero lo hace extendiéndola más y, por tanto, vuelve a circunscribirse a la tradición nacionalista.

Las antropólogas Glick, Basch y Szanton, en un trabajo publicado en 1992, utilizaron el término transnacional para poner de relieve la emergencia de espacios sociales transnacionales que relacionan las sociedades de origen y de destino, y que permiten el mantenimiento y el refuerzo de los lazos con los lugares de procedencia generando, de esta forma, comunidades cuya característica principal es su desterritorialización; es decir, que no tienen un espacio físico concreto. Estos espacios sociales transnacionales se representan como espacios telemáticos, que permiten la comunicación y la relación de forma electrónica, a distancia y en red, en los cuales se crean comunidades transnacionales (Faist, 1999; Pries, 1999) formadas por migrantes de todo el mundo cuyas características comunes les unen entre sí, y cuyo medio de interconexión no es otro que el espacio electrónico, también llamado telemático, digital o virtual.

El prefijo trans, que significa al otro lado de o a través de, no supone una ruptura con el enfoque nacional, sino que lo subraya para resaltar la amplitud y la extensión de la nación más allá de su contenedor habitual, es decir, de las fronteras de la nación. Y este deseo de los países de origen de construir un sentimiento de pertenencia nacional más allá de las fronteras, implicando a las personas que ya no están, que han dejado el topos nacional, es un paso más en la configuración de la comunidad imaginada definida por Benedict Anderson. Según sus palabras:

Es imaginada porque aún los miembros de la nación más pequeña no conocerán jamás a la mayoría de sus compatriotas, no los verán ni oirán siquiera hablar de ellos, pero en la mente de cada uno vive la imagen de su comunión (Anderson, 1993, p. 23).

La creación de pertenencia nacional más allá del territorio implica una ampliación del Estado al encarnarse no sólo en la geografía nacional sino en todas las personas que han abandonado el lugar pero que se les presupone e incita al mantenimiento de la pertenencia colectiva al Estado-nación de origen. El ejercicio de imaginar la comunidad

Para citar este artículo:
González, Y. (junio, 2012). Migración transnacional colombiana y estrategias políticas online. Reproducción de la comunidad a través de la vinculación transnacional. Ánfora, 19 (32), 69 - 90. Universidad Autónoma de Manizales. ISSN 0121-6538. 
nacional al que se le invita a los migrantes, no dista mucho del ejercicio realizado por cualquier persona que se siente miembro de alguna comunidad ${ }^{1}$. La pretendida encarnación de la nación se pone en evidencia en las campañas de propaganda y las políticas activas de vinculación online de las comunidades de emigrantes que se analizará más adelante en el material visual encontrado en la web RedEsColombia.

Utilizar el concepto de red social, y en concreto en el ámbito telemático, para los estudios transnacionales resulta especialmente fructífero en la medida en que estas prácticas se fundamentan y se mantienen invariables gracias a los recursos sociales que los individuos poseen, en defecto de los económicos o políticos. Vertovec expresa la validez del análisis de redes para el estudio de las actividades transnacionales de la siguiente manera: "social network analysis has operationalized many terms and concepts that researchers of transnacional social formations would do well to bear in mind when collecting, analyzing and describing data" (Vertovec, 2003, p. 647).

\section{Migración en Colombia. Aproximación a su contexto migratorio y a su situación actual}

El caso de Colombia es significativo cuando se habla de la creación de estructuras online para la conexión de los migrantes. El portal virtual lanzado por el gobierno se encuentra dentro de un proyecto de política migratoria mucho más amplio como es el Programa Colombia Nos Une que se explica más adelante.

En primer lugar, se considera necesario presentar, a grandes rasgos, la historia y situación migratoria de Colombia, de manera que se vislumbre la realidad objetiva por la que el país transita y los condicionantes cuantitativos y cualitativos que llevan al gobierno colombiano a establecer una política integral de migraciones, más allá de otras motivaciones que podrían ser más subjetivas, que están detrás de la acción gubernamental y las cuales se retoman adelante en este artículo.

Según datos del Censo Nacional de Población del año 2005², Colombia tiene una población de 3.378.345 personas que residen en el exterior, lo cual supone algo más del $8 \%$ del total de su población residente en Colombia. Sin embargo, la tarea de calcular la magnitud y el destino de los emigrantes colombianos no es fácil, a pesar de los incontables esfuerzos, en especial de parte del mundo académico, de realizar estimaciones y comparar datos tomados de distintas fuentes. Si bien es cierto que el Estado colombiano, a través del Departamento Administrativo de Seguridad (DAS), lleva un control

1 Como apunta Anderson, considerar la nación como una invención dista mucho de considerarla una fabricación o una falsedad, sino que tiene que ver más bien con una creación o una imaginación lo cual no presupone la existencia de comunidades verdaderas diferentes a las imaginadas (1993).

2 El último realizado hasta el momento. Departamento Administrativo Nacional de Estadística (DANE).

Para citar este artículo:
González, Y. (junio, 2012). Migración transnacional colombiana y estrategias políticas online. Reproducción de la comunidad a través de la vinculación transnacional. Ánfora, 19 (32), 69 - 90. Universidad Autónoma de Manizales. ISSN 0121-6538. 
exhaustivo de las entradas y salidas de personas del país, este tipo de registros no dan cuenta de personas emigradas sino de movimientos fronterizos de entrada y salida.

Otra de las fuentes a las que se suele atender es el programa de registro consular del Ministerio de Relaciones Exteriores (MRE) que, como es sabido, no recoge la realidad emigratoria de la población colombiana en los países de destino y que ofrece cifras muy por debajo de las estimaciones hechas, las cuales alcanzan para el año 2005 los 5 millones de personas que viven en el exterior de Colombia ${ }^{1}$.

Se suele hablar de tres oleadas de emigración en diferentes momentos históricos y por diferentes causas. La primera ocurrió durante la década de los sesenta y principios de los setenta y tuvo como destinos principales a Estados Unidos y Venezuela, mientras que la segunda se produjo durante los ochenta, cuando se incrementó la emigración hacia los mismos destinos que en la anterior pero influida esta vez por las redes sociales que los ya emigrados habían formado en esos destinos. Por último, la tercera oleada tuvo lugar a partir de mediados de los noventa y se intensificó a partir de 2000, y con un cambio esencial en el destino, pues, por primera vez y a partir de ese momento, España es el país que mayor número de colombianos y colombianas recibe (29,1\%). En esta tercera etapa, además de España, siguen siendo claves los destinos tradicionales como Estados Unidos $(27 \%)$ o los países fronterizos como Venezuela $(17,3 \%)$ o Ecuador $(4,4 \%)^{2}$.

\section{Gráfico 1. Evolución del total de emigrantes colombianos 1960 - 2005}

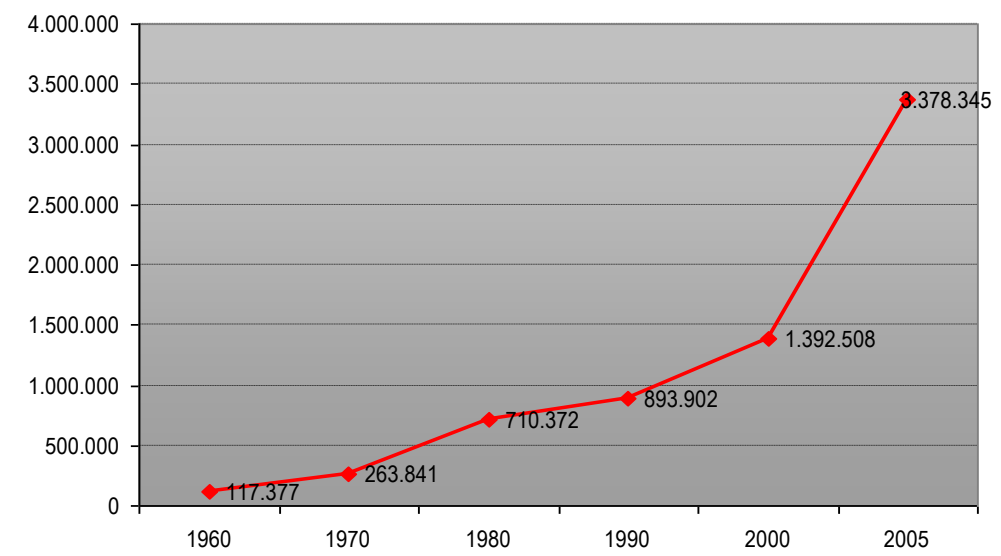

Fuente: Banco de datos CELADE y censos de población del DANE

\footnotetext{
1 Estas estimaciones de Ministerio de Relaciones Exteriores se realizan teniendo en cuenta los flujos migratorios pasados, las cifras de otros países similares a Colombia y los factores que indiquen la presencia de colombianos en cada uno de los países extranjeros.

2 Datos extraídos de Garay y Medina (2008).
} 
Si bien es cierto que estas tres etapas tienen relevancia por ser momentos de auge de los flujos migratorios hacia el exterior de Colombia, si se tiene en cuenta la magnitud de dichas salidas, queda de manifiesto que el boom emigratorio de la última época no tiene comparación cuantitativa con las dos anteriores.

\section{Política migratoria colombiana. El programa Colombia Nos Une}

En este contexto, y durante la segunda parte de la década de los noventa, el Estado colombiano fue dándose cuenta progresivamente de cuál era la situación migratoria del país, cuál era el volumen de nacionales que residían fuera y, sobre todo, cómo se presentaba la tendencia emigratoria hacia el futuro, con la más que segura salida de Colombia de grandes grupos de población que profundizaría esta realidad. Por lo tanto, el gobierno vio la necesidad de que la población colombiana en el exterior recibiera una atención integral del Estado y, en consecuencia, comenzó a incluir en sus políticas públicas la temática migratoria. Es en el Plan Nacional de Desarrollo 2003 - 2006 cuando se recoge la idea de lanzar una política global relacionada con los diversos grupos que conformaban la comunidad colombiana en el exterior, con el propósito explícito de estimular los vínculos con el país y hacerlos objeto de políticas públicas.

Con el fin de consolidar estas estrategias, se llevó a cabo en junio de 2003 el seminario "Migración Internacional Colombiana y Formación de Comunidades Transnacionales" en el cual se propició la reflexión y la sensibilización frente al fenómeno migratorio colombiano de la última década. En el marco de este seminario, se dio inicio al Programa Colombia Nos Une, y se propició entre los sectores público, privado y académico, un ejercicio de reflexión alrededor de nueve áreas temáticas, cuyas conclusiones y recomendaciones fueron la base para la puesta en marcha del programa.

El Programa Colombia Nos Une se fundamenta en la presunción de la existencia de relaciones transnacionales entre comunidades de colombianos. Según el proyecto, "el transnacionalismo busca comprender los procesos migratorios mediante los vínculos familiares, las relaciones sociales, económicas, religiosas o políticas que mantienen los emigrantes con Colombia y las redes sociales que surgen mediante este proceso e involucran a un número considerable de individuos tanto en los Estados receptores como en el país de origen" (Colombia Nos Une, 2011).

Colombia Nos Une pretende incrementar y hacer más fuertes los lazos de las comunidades colombianas que residen en el extranjero, reconociéndolas como parte esencial de la nación colombiana y, por tanto, teniéndolas como objeto de política pública. El programa incluye entre sus objetivos impulsar el establecimiento de vínculos entre las comunidades colombianas en el exterior y el país, identificar los intereses y las necesidades de los colombianos en el exterior, gestionar mecanismos orientados a mejorar sus condiciones de vida en los lugares de residencia y propiciar encuentros con las comunidades colombianas que viven fuera del país.

Para citar este artículo:
González, Y. (junio, 2012). Migración transnacional colombiana y estrategias políticas online. Reproducción de la comunidad a través de la vinculación transnacional. Ánfora, 19 (32), 69 - 90. Universidad Autónoma de Manizales. ISSN 0121-6538. 
En cuanto a la plataforma web nacida de Colombia Nos Une, en primer lugar, es necesario explicar que el Portal RedEsColombia es un espacio virtual abierto, accesible e integrador, teóricamente dirigido a las personas que estén interesadas en Colombia. Sin embargo, la plataforma fue inicialmente concebida para vincular a las personas emigradas con su país de origen, siendo la población emigrada la población objetivo del programa. Es por ello que, aunque utiliza la estrategia de poner en contacto a personas individuales, bien sean residentes en el exterior o bien en Colombia, su objetivo fundamental es relacionar a dichas personas con el país y, por tanto, con el Estado y su gobierno. Pretende dar a conocer una posición de cercanía de las instituciones con los migrantes, sus familias y los entornos de origen. Esta plataforma tecnológica es un punto de encuentro que tiene como una de sus finalidades el fortalecimiento del capital social entre los colombianos, proporcionándoles un espacio social de acción e interacción dinámico, a distancia y participativo, como estrategia de aprovechamiento de su potencial contribución al desarrollo del país.

La pregunta fundamental tiene que ver con el porqué es en este momento de la historia migratoria de Colombia, cuando el Estado pone en marcha políticas activas de vinculación de sus nacionales residentes en otros países. Hay, desde luego, algunos factores más o menos evidentes y explicitados en los discursos oficiales que tienen que ver, por un lado, con el desarrollo de un Estado más consciente de la problemática que puede generar la movilidad humana en los términos en que se ha producido para el caso colombiano, con una estimación de cerca de 5 millones de nacionales en el extranjero y, por otro lado, con la preocupación que la situación de éstos puede generar al gobierno en el poder, tanto de manera objetiva y en respuesta a su obligación de protección de sus nacionales, independientemente de donde se encuentren residiendo, como, por otra parte, motivada por razones electoralistas consciente de la gran cantidad de votos que suponen las personas emigrantes.

Sin embargo, hay otro tipo de factores que pueden quedar relegados en el discurso oficial, y por tanto invisibilizados. Estos elementos tienen que ver con el momento migratorio que está viviendo el país o, por decirlo más exactamente, con la etapa migratoria de los colombianos y las colombianas en el exterior. Es decir, es en la actualidad cuando se está produciendo el asentamiento con visos de ser definitivo de las personas emigradas durante la oleada de salida de finales de los años noventa y principios de la década del 2000.

Diversos estudios para el caso estadounidense y en relación con diferentes comunidades nacionales (colombianos, dominicanos y mexicanos), han demostrado que son los grupos de migrantes con niveles altos de asentamiento en el destino los que participan más de actividades transnacionales de todo tipo, tanto económicas, como sociales, cívicas y de participación política (Portes, Haller and Guarnizo, 2002; Guarnizo, Portes and Haller, 2003; Portes, Escobar y Walton, 2007). 
Es en este momento del proyecto migratorio de la inmensa mayoría de los colombianos y colombianas que residen en el exterior del país, en el que los gobiernos de origen ven mayores necesidades de actuaciones concretas que sigan uniendo a los migrantes con sus países de origen. La importancia estructural que tienen actividades transnacionales como el envío de remesas, la inversión en las localidades de origen en terrenos o empresas y, sobre todo, en vivienda, así como las escasas pero significativas actividades cívicas y filantrópicas, es causa más que suficiente para impulsar a los gobiernos a realizar políticas encaminadas a mantener la vinculación de sus expatriados. Los países de origen necesitan conservar la lealtad de su emigrados e incluso potenciar actividades como las remesas y las inversiones, que se ven en riesgo debido al asentamiento semidefinitivo y la integración efectiva en las sociedades de destino, lo cual puede hacer tambalear el compromiso personal con la escasa familia que queda en el origen (tras haber realizado la reagrupación de la familiar más cercana) y con el país en general.

Se puede llegar a pensar, como señala Portes et al. (2007), que el transnacionalismo inmigrante es un efecto de dichas iniciativas políticas, o sea que se producen como reacción a ellas. Sin embargo, la evidencia empírica indica que las prácticas transnacionales surgieron por iniciativa de los propios migrantes y que sus gobiernos se sumaron a este empeño sólo cuando se puso de manifiesto su importancia y sus potencialidades económicas para el desarrollo del país. En este sentido, se podría decir que el discurso que despliegan los gobiernos de origen sobre la vinculación de sus emigrantes, es un discurso históricamente situado, que se origina a través de la constatación de una realidad migratoria que ha evolucionado hacia una estabilización en destino de la población migrante, la cual, además, viene emprendiendo iniciativas de relación transnacional con el contexto de origen. De todas formas, sería ingenuo pensar que las acciones gubernamentales no están en cierta medida redirigiendo y orientando la naturaleza y la forma en que se está produciendo en la actualidad el vínculo transnacional entre los emigrados y sus sociedades de origen. De ahí la importancia y la necesidad de analizar este tipo de procesos ya que pueden influir la manera en que se desarrolla el transnacionalismo migrante.

\section{Metodología}

El análisis de RedEsColombia se ha planteado mediante la realización de una exploración cuantitativa de la evolución y situación del Portal RedEsColombia y de las redes creadas desde el nacimiento de la plataforma hasta junio de 2010, en especial, en referencia al tipo de redes, su volumen, la localización de sus miembros, etc. Esto permite ver en cierta medida la extensión y el desarrollo de las redes, y evaluar parcialmente el éxito del Portal. Con este análisis cuantitativo, se refleja una imagen más o menos nítida del estado actual de las redes que se han formado dentro de esta iniciativa

78 y de cómo están utilizando el Portal los usuarios individuales o colectivos.

Para citar este artículo:
González, Y. (junio, 2012). Migración transnacional colombiana y estrategias políticas online. Reproducción de la comunidad a través de la vinculación transnacional. Ánfora, 19 (32), 69 - 90. Universidad Autónoma de Manizales. ISSN 0121-6538. 
Debido al especial valor simbólico que lo visual tiene en el contexto virtual, se ha recogido, además, la información visual contenida en la web a través de videos, tours virtuales, campañas publicitarias, noticias y eventos destacados. Se ha realizado así un análisis cualitativo y discursivo de dichos materiales, que se distancian de ser documentos oficiales con cariz de justificación política de la iniciativa, y que pasa a ser una campaña de comunicación e información que tiene como objetivo directo a la población colombiana que se acerque al Portal o que pretenda acercarse a otros colombianos.

En este apartado metodológico es necesario explicar que el análisis cuantitativo y cualitativo de las políticas activas de migración colombianas, y en concreto de RedEsColombia, se ha realizado desde la perspectiva de destino, es decir, enmarcada en una investigación diseñada bajo el enfoque de un país de recepción de emigración colombiana como es España y de una investigadora no migrante.

El diseño de la exploración analítica realizada subraya una realidad hipotetizada en los objetivos de la investigación y que busca estudiar la incidencia que las políticas públicas respecto al fenómeno migratorio tienen sobre el desarrollo y la evolución de la relación migratoria entre personas y comunidades.

\section{Resultados}

\section{Análisis de la Plataforma RedEsColombia. Redes y usuarios. ¿Éxito cuantitativo?}

Los datos cuantitativos utilizados a continuación se refieren a información secundaria procedente de las estadísticas y el seguimiento que realiza el propio Programa Colombia Nos Une en colaboración con la OIM. A la luz de las estadísticas, es necesario advertir que el gran volumen de población registrada ${ }^{1}$ en el Portal reside en Colombia, y no en el extranjero. A pesar de que RedEsColombia tiene como población diana a los emigrados, más de la mitad de usuarios no pertenecen a este grupo de población.

Pueden existir varias hipótesis para explicar esta situación, que se podría decir es contraria a los objetivos del programa. En primer lugar, hay que tener en cuenta las ansias de la población colombiana residente en el país de un espacio para compartir, ya no con quienes se fueron del país, sino con los que aún siguen dentro y que se encuentran a busca de oportunidades de cualquier tipo (bien de desarrollo en Colombia o bien de posibilidades en el exterior). Este espacio compartido es quizá un deseo más fuerte y una necesidad mayor para los colombianos residentes en Colombia que para los que ya se han establecido en otros lugares.

1 No se debe confundir el hecho de estar registrado en una red con el de ser usuario activo y frecuente de la misma. 
Sin embargo, es necesario introducir en el análisis otras variables que afectan también a la situación social y a los deseos de las personas emigradas, para comprender por qué su vinculación a través de RedEsColombia es inferior a la de los no emigrados. La manera en que las personas emigradas conciben su relación con el país de origen, sus necesidades de vincularse con él y el momento del proceso de integración social en el que se encuentran en destino, así como el tipo de integración del que son objeto-sujeto, es posible que influyan de manera significativa en el hecho de utilizar esta plataforma tecnológica y de cómo hacerlo.

Salvando esta diferencia entre la afiliación entre colombianos emigrados y no emigrados, es un hecho explicitado que RedEsColombia está albergando una gran cantidad de usuarios, que, en términos cuantitativos, no para de crecer y ha logrado hacer saber de su existencia a un volumen considerable de su población, en un corto tiempo. Como se observa en el Gráfico 2, a mediados de 2010 hay más de 38.000 usuarios registrados de los cuales el 53,6\% reside en Colombia. El crecimiento ha sido realmente espectacular, si se tiene en cuenta que en enero de 2008 sólo ascendía a 1.544 usuarios. El incremento porcentual en apenas dos años y medio ha sido de más del $2.300 \%$. Sin embargo, el crecimiento en el último semestre ha sido más moderado y puede ser el primer indicio de la ralentización del aumento cuantitativo de RedEsColombia.

\section{Gráfico 2. Evolución usuarios registrados en RedEsColombia. Enero 2008 - Junio 2010}

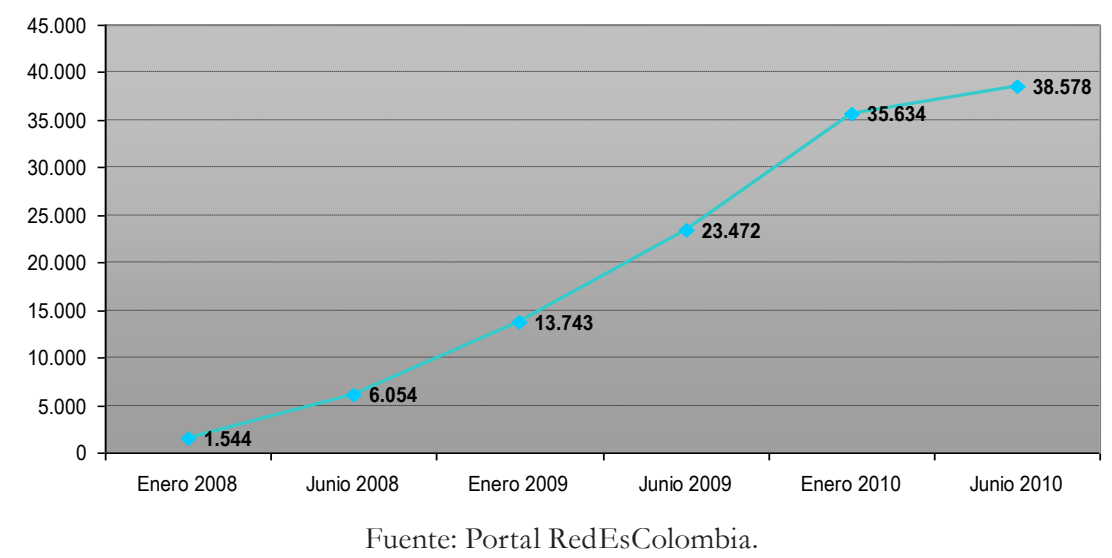

Así mismo, en lo que respecta al número de redes, el crecimiento ha sido también muy importante, pasando de 116 en enero de 2008 a 1.044 en junio de 2010. De la comparación de ambos volúmenes se desprende que la inmensa mayoría de los usuarios de RedEsColombia no son creadores de redes propiamente dichas, sino que únicamente tienen perfil propio y participan en la red global y pertenecen a una o varias redes integradas en

80 la plataforma virtual, siendo sólo el 2,7\% los que han creado una red propia. 


\section{Gráfico. 3. Evolución redes registradas en RedEsColombia. Enero 2008 - Junio 2010}

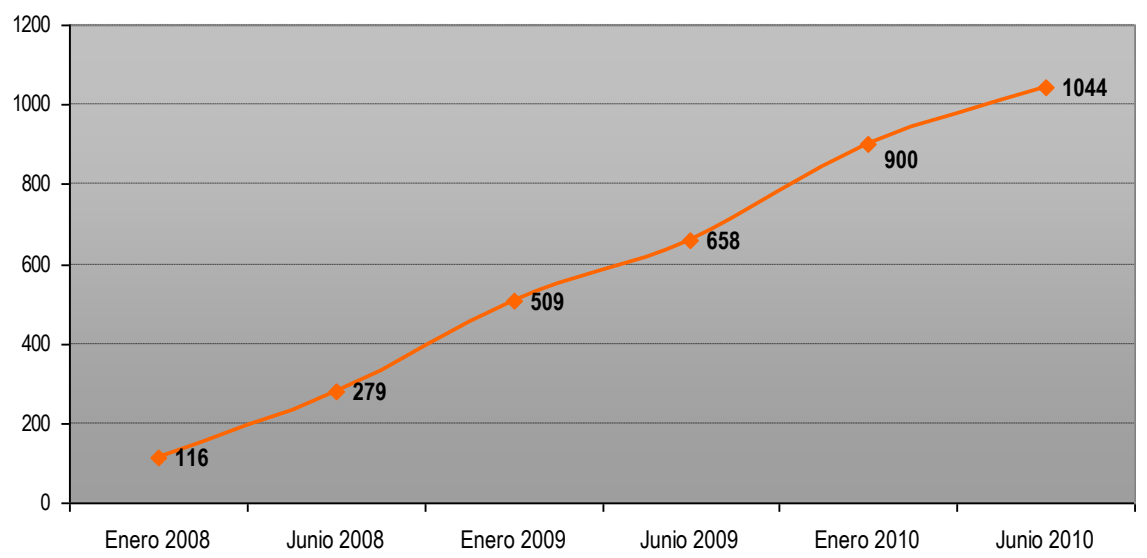

Fuente: Portal RedEsColombia.

Tras observar el crecimiento del número de usuarios y de redes, es necesario ver si el desarrollo cualitativo ha sido similar y se puede hablar de significatividad en cuanto a actividad, relaciones y contenidos. Analizando los datos que indican cuáles son las redes con mayor número de miembros y cuál es el motivo o iniciativa desde la que surgen, se observa una escasez de redes que presentan un volumen sustancial de miembros y, sin embargo, la inmensa mayoría (98\%) de las redes creadas hasta el momento no superan los 100 miembros, y gran parte de ellas son redes realmente pequeñas, de uno o dos miembros. Por otra parte, la red con más miembros: Colombianos apasionados, acoge al $4 \%$ del total de usuarios registrados en RedEsColombia, que ha sido creada y es administrada por Colombia es Pasión, una iniciativa del Gobierno colombiano a través de la cual se pretende crear una estrategia de Imagen País mediante la que se "busca mejorar la percepción del país en el exterior, que genere conocimiento y confianza para alcanzar más y mejores oportunidades en materia comercial, inversión y turismo"1.

Observando el ranking de redes más populares, se observa cómo las que tienen las primeras posiciones son redes creadas por organismos públicos, administradas por ellos y que pretenden dar a conocer sus iniciativas y sus propuestas. La segunda red más importante es Tecnoparque Colombia, un programa del Servicio Nacional de Aprendizaje (SENA) dedicado a la "promoción del talento humano, con compromiso hacia el desarrollo tecnológico, la innovación y el emprendimiento en Colombia.” Y la tercera es la perteneciente al Programa Colombia Nos Une, es decir, al Ministerio de Relaciones Exteriores.

1 Tomado de www.colombiaespasión.com. 
Tabla 1. Redes más populares y proporción de miembros sobre el total de usuarios. 3/06/2010

\begin{tabular}{|l|c|c|}
\hline \multicolumn{1}{|c|}{ Nombre de la red } & $\mathbf{N}^{\mathbf{0}}$ miembros & $\begin{array}{c}\text { \% sobre total } \\
\text { usuarios }\end{array}$ \\
\hline Colombianos Apasionados & 1.567 & 4,1 \\
\hline Tecnoparque Colombia & 1.003 & 2,6 \\
\hline Colombia Nos Une & 1.028 & 2,7 \\
\hline Colombianos en el Reino Unido & 388 & 1,0 \\
\hline Consulado de Colombia en Argentina & 345 & 0,9 \\
\hline Consulado General de Colombia en Chile & 358 & 0,9 \\
\hline Universidad Distrital Francisco José de Caldas & 226 & 0,6 \\
\hline Consulado General de Colombia en París & 247 & 0,6 \\
\hline Conexión Colombia & 264 & 0,7 \\
\hline Consulado de Colombia en Madrid & 214 & 0,6 \\
\hline $\begin{array}{l}\text { Red de estudiantes y profesionales colombianos } \\
\text { en el exterior }\end{array}$ & 224 & 0,6 \\
\hline Club Networking TIC & 177 & 0,5 \\
\hline Con Uribe en la lucha contra las FARC & 174 & 0,5 \\
\hline Programa Ondas & 135 & 0,4 \\
\hline Asociación de colombianos en Chile & 143 & 0,4 \\
\hline Diásporas digitales & 129 & 0,3 \\
\hline No al secuestro & 111 & 0,3 \\
\hline Consulado General de Colombia en Barcelona & 149 & 0,4 \\
\hline Resto redes & 31.270 & 82,0 \\
\hline Total usuarios & $\mathbf{3 8 . 1 5 2}$ & $\mathbf{1 0 0 , 0}$ \\
\hline
\end{tabular}

Fuente: Portal RedEsColombia

El resto de redes con mayor número de miembros pertenecen principalmente a Consulados o Embajadas de Colombia en otros países, como Argentina, Chile, Francia o España. Así mismo, existen otras redes dedicadas al conocimiento científico, como la Red de estudiantes y profesionales colombianos en el exterior, la de la Universidad Distrital Francisco José de Caldas o la red Programa Ondas, además de otras dedicadas al debate de temas políticos del país, como Con Uribe en la lucha contra las FARC o No al secuestro.

De todo ello, se puede concluir que la iniciativa pública es la que genera las redes más exitosas, mientras que la privada, a pesar de crear un número considerable de nue82 vos nodos, no consigue producir actividad a partir de ellos ni atraer a más personas ha- 
cia los intereses que persiguen. La naturaleza institucional del Portal RedEsColombia parece que influye fuertemente en el tipo de redes que se crean en su interior y en especial en los diferentes niveles de popularidad entre las redes oficiales-gubernamentales, es decir, creadas por organismos públicos o dependientes del Estado, y las redes informales o privadas, es decir, creadas por personas naturales con intereses particulares.

Según el tipo de red, el Portal las clasifica en 17 grupos, que para simplificar se congregan aquí en 7 categorías: Cultura y ocio, Asociaciones de colombianos/as en el exterior, Negocio e inversión, Sociedad y Política, Educación y conocimiento, Colombia es Pasión, y Otros.

Como se observa en el Gráfico 4, 23\% del total de las redes se agrupan en la categoría Sociedad y Política, mientras que el 18\% lo hace en Cultura y Ocio. Estas dos categorías, junto con Colombia es Pasión (5,3\%), giran en torno a redes interesadas en asuntos internos o cuya creación ha sido motivada por usuarios residentes en Colombia, y en las cuales la temática migratoria no ejerce una influencia determinante.

\section{Gráfico 4. Distribución porcentual del número de redes por tipo de red. $3 / 06 / 2010$}

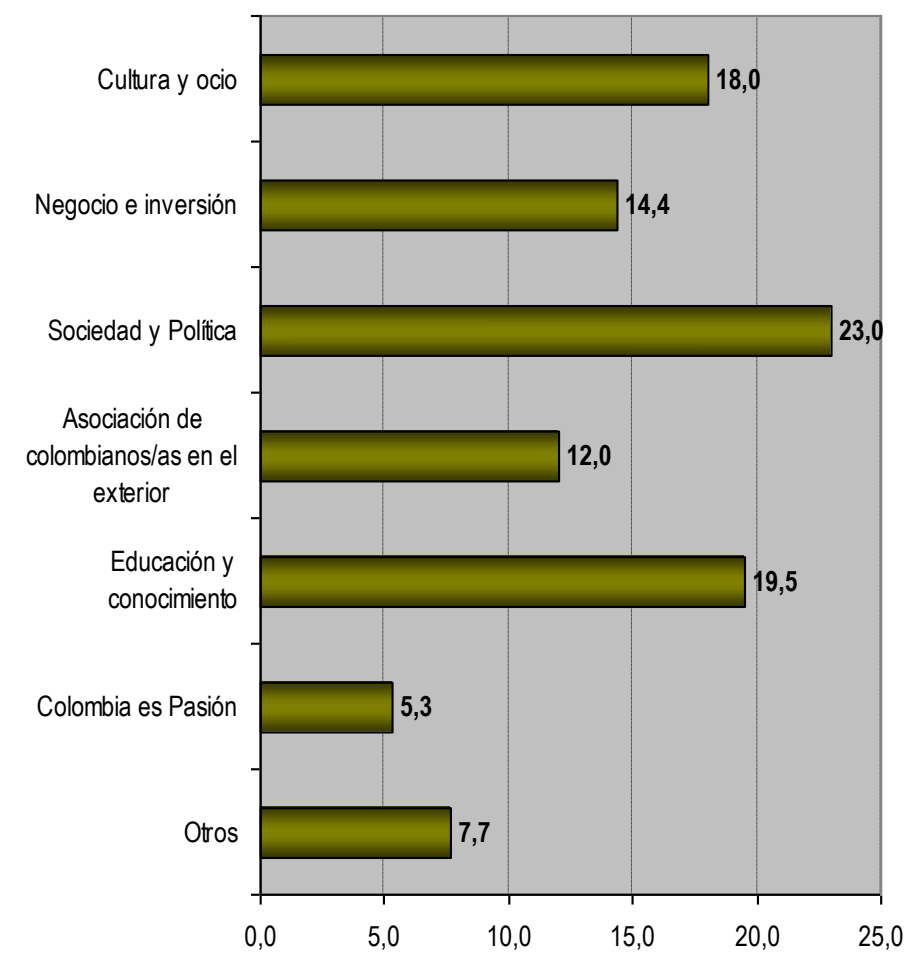

Fuente: Portal RedEsColombia 
Por otro lado, hay otros tipos de redes como las clasificadas dentro de Asociaciones de colombianos y colombianas en el exterior, que representan el 12\% del total, que tienen relación directa con la vinculación de personas emigradas con el país, lo cual, recordemos, es el principal objetivo del Portal RedEsColombia. Además, las categorías Negocio e Inversión, con el 14,4\% y Educación y Conocimiento, con el 19,5\%, son también reflejo del espíritu con que se crea la plataforma, es decir, promover al aprovechamiento del potencial de contribución de los migrantes al desarrollo del país, bien a través de transferencia del conocimiento científico adquirido en el extranjero o bien con el retorno de dicho capital humano o con el fomento de la creación de iniciativas empresariales y de inversión en Colombia.

Un análisis que dé un paso más en la investigación sobre los factores que influyen o condicionan la membresía en esta red social institucional, necesitaría del estudio de otros datos que no son objeto de las estadísticas que el Portal ofrece . La caracterización somera de las redes y de los usuarios de RedEsColombia es sólo un primer peldaño en la búsqueda de respuestas y una buena fuente de generación de hipótesis que aludan a las causas, las motivaciones y las formas por las que se produce la inserción en estas redes, en relación con las consecuencias que dicha inserción tiene sobre la práctica transnacional de los migrantes, tanto en la manera en que se produce como en el alcance y las tipologías que desarrollan.

\section{Fundamentos simbólicos de la política de vinculación de la comunidad colombiana emigrada}

Además de estadísticas sobre usuarios y redes, RedEsColombia ofrece mensajes claros y concisos a través de la información visual contenida en la web, que explicitan los fundamentos sobre los que gira la política migratoria colombiana. Estos mensajes son difundidos a través de pequeños testimonios, de reportajes y de canciones que, en algunos casos, son lanzados por personas emigradas en diferentes lugares del mundo (Berlín, Madrid, Toronto, Seúl, Buenos Aires y Lisboa) y también por personalidades colombianas con éxito internacional como el cantante Juanes, el cafetero Juan Valdés o el director de cine Sergio Cabrera, entre otros. A continuación, se presentan los ejes sobre los que se basa este discurso.

En primer lugar, y como objetivo más claro presente en cada uno de los textos audiovisuales y escritos del Portal, aparece el deseo de formar Comunidad, tal y como es definida por Benedict Anderson (1993) y explicada arriba, y que pretende prolongar o renovar el sentimiento de pertenencia nacional de los colombianos y las colombianas que viven en el exterior. La referencia constante a la comunidad colombiana evoca un renacimiento de ser y sentirse colombiano o colombiana, con la intención de evitar el olvido de la colectividad, recordando las características primigenias que les unen a

84 Colombia y que, de alguna forma, les constriñe inevitablemente:

Para citar este artículo:
González, Y. (junio, 2012). Migración transnacional colombiana y estrategias políticas online. Reproducción de la comunidad a través de la vinculación transnacional. Ánfora, 19 (32), 69 - 90. Universidad Autónoma de Manizales. ISSN 0121-6538. 
Que no se olviden de su tierra, de sus costumbres y de sus raíces, que las tengan siempre presentes. Y les digo que tienen un vínculo con Colombia el cual no se puede borrar ni quitar (...) Desde donde estás, tu nos sentirás, unido de tus costumbres, unido siempre estarás (Video 2, Portal RedEsColombia).

Búsqueda de un estatus de ciudadanía que vaya más allá de las fronteras del Estado-nación, que se convierta en transnacional y no sólo en lo concerniente a los derechos sino también a las obligaciones como colombianos y colombianas, incluso mucho más allá de la nacionalidad. Se hace énfasis en la membresía a la comunidad como mecanismo de comunicación intergrupal, con otros colectivos nacionales o en otros lugares del mundo ("Mostrar al mundo que somos colombianos") y como estrategia de comunicación intragrupal que facilite la unión a través de intereses compartidos.

Sus intereses pueden ser los mismos de muchos otros colombianos alrededor del mundo. Aquí (en RedEsColombia) usted encuentra las herramientas para relacionarse con ellos, sin importar si viven en la misma ciudad o en diferentes continentes (...) sus historias, intereses y opiniones son importantes para otros colombianos (Tour Virtual, Portal RedEsColombia).

El sentimiento de comunidad y la pasión por Colombia es un mensaje que se repite constantemente, como estrategia de unidad, como nexo común entre colombianos y colombianas, sin importar la ideología política, el estatus económico, las diferencias culturales ni, por supuesto, la condición de migrante. Las campañas de imagen que se han puesto en marcha en los últimos años se dirigen hacia la puesta en valor del sentimiento de unidad nacional, con especial énfasis en el orgullo y la pasión de ser colombiano. Y esta campaña se intensifica si cabe aún más para el caso de la población emigrada.

Otro de los ejes sobre los que gira el discurso en el Portal es el que se refiere a la internacionalización de Colombia, al deseo de mejorar la imagen internacional del país por los cauces oficiales y también a través de la unidad en el sentir de la población colombiana. La Imagen-País Colombia es Pasión pone sus esfuerzos en mostrar al mundo un valor diferencial. Esta estrategia de mejora de la imagen del país, tiene a los migrantes en uno de sus focos de atención. La vida y el trabajo que los colombianos y las colombianas llevan a cabo a lo largo del mundo es el mayor exponente de lo que es Colombia, traspasando por tanto una responsabilidad al migrante de ser portavoz y abanderado de la imagen del país en el exterior.

Los colombianos fuera de Colombia somos como una semilla importante de nuestro país fuera y tenemos una responsabilidad muy grande en que esa semilla germine bien y que sea de utilidad tanto para el país que nos acoge como para nuestro país de origen que es Colombia. Y eso es algo que no se consigue si no se tiene una voluntad, yo creo que es importante que los colombianos tengamos la

Para citar este artículo:
González, Y. (junio, 2012). Migración transnacional colombiana y estrategias políticas online. Reproducción de la comunidad a través de la vinculación transnacional. Ánfora, 19 (32), 69 - 90. Universidad Autónoma de Manizales. ISSN 0121-6538. 
voluntad de hacer grande a nuestra patria y hacerla grande también en la patria que nos acoge (Video 1, Portal RedEsColombia).

El destacado papel que el Gobierno colombiano otorga a sus migrantes nace del convencimiento de que suponen un capital humano muy valioso para el país, pero del que es difícil su aprovechamiento en la distancia. RedEsColombia pretende darle valor este capital humano y se presenta como un cauce para su fortalecimiento y su recuperación. Dos estrategias surgen en el discurso recogido: por un lado, fortalecer el capital humano que se encuentra dentro y fuera del país a través del intercambio de información y conocimiento y, por el otro lado, recuperar ese capital a través de propiciar un espacio para la creación de redes y proyectos que se dirijan a mejorar el desarrollo del país en los que se puedan involucrar los emigrados. Esta recuperación del capital humano es un retorno de los cerebros fugados pero sin necesidad del retorno físico al país. Algunas de las noticias recientes que se pueden ver en el Portal hacen referencia a la recuperación del capital humano: "Colombia busca reanudar lazos con sus cerebros fugados ¿Podrá Colombia sacar ventaja de sus cerebros fugados? Sí, gracias al Internet” (Noticia 1, Fecha publicación: 09/04/2010, Portal RedEsColombia). La Red se presenta como el espacio idóneo en el que...

Se transmite, intercambia y comparte información, conocimiento, competencias, saberes y experiencias. [...] Usted puede compartir recursos disponibles en Internet así como sus textos especializados sobre los temas de interés para su red o puede encontrar los aportes de otros autores (Tour Virtual, Portal RedEsColombia).

Al mismo tiempo, y aunque no se presenta como uno de los objetivos de la Red, el retorno físico es alentado y figura como posibilidad real dentro del proceso migratorio colombiano. Las canciones contenidas en varios de los videos del Portal aluden al deseo de que vuelvan los que se han ido:

Dijeron volver un día [...] ¿Cuándo volverán los que son de acá? (Video 2, Portal RedEsColombia). Estamos aquí porque queremos unirnos a los colombianos que no nos pueden acompañar en este país [...] esta canción habla de las ganas que tenemos de que algún día estos que están por fuera, estos coterraneos, estos parseros vuelvan, vuelvan pronto (Video 3, PortalRedEsColombia).

Pero, sin duda, el concepto clave alrededor del que gira el discurso implícito y explícito sobre RedEsColombia, y en general en el Programa Colombia Nos Une, es el referido al Desarrollo, con mayúscula, del país. Bajo este objetivo, se puede agrupar el resto de los ejes nombrados, porque, aunque se produzcan en diferentes direcciones o por distintos senderos, la pretensión más clara del gobierno colombiano al implementar este tipo de iniciativas políticas es contribuir al desarrollo del país, con la ayuda de las personas emigradas a través de diferentes mecanismos, 86 tanto indirectos (como puede ser la mejora de la imagen del país) como directos

Para citar este artículo:
González, Y. (junio, 2012). Migración transnacional colombiana y estrategias políticas online. Reproducción de la comunidad a través de la vinculación transnacional. Ánfora, 19 (32), 69 - 90. Universidad Autónoma de Manizales. ISSN 0121-6538. 
(como el fortalecimiento del sentimiento de comunidad y la vinculación-responsabilidad con el país, o la recuperación del capital humano). El concepto de Desarrollo aparece constantemente en la explicación de lo que es RedEsColombia, tanto en la forma literal del término, como de forma tácita en los textos de audio y video que se analizan aquí. "Lo que más me gusta de RedEsColombia es que es más que un entretenimiento, es una herramienta en la cual podemos encontrar proyectos para buscar un impulso al desarrollo de nuestro país" (Video 1, Portal RedEsColombia).

El Gobierno colombiano trabaja en una línea comunitarista, poniendo de manifiesto la necesidad de ayudar sólo a la familia o los amigos que se dejaron en el país, y también al conjunto del pueblo colombiano. Y con este objetivo le presenta al migrante esta oportunidad, que se convierte en una responsabilidad hacia el país al que siguen perteneciendo. "Si aspiras a quedarte lejos [...] construye poco a poquito lo que empezaron tus viejos" "Si te vas y te quedas lejos, tu casa ha de ser primero" (Video 2, Portal RedEsColombia).

En este punto se enlazan perfectamente el objetivo de promover el sentimiento de comunidad entre los emigrados y el de que éstos contribuyan a su desarrollo. $\mathrm{El}$ primero es casi condición sine qua non para que se produzca el segundo. Se pone de relieve que el gobierno no olvida a sus emigrantes y también piensa en el futuro de su población, tanto de la emigrada como de la no emigrada. "Aquí está el futuro del mundo, de nuestros hijos, pa' nuestros nietos" "[Los migrantes] se llevan su alma y su vida dejándoles el recuerdo a su pueblo colombiano que también piensa en el progreso" (Video 2, Portal RedEsColombia). Este objetivo desarrollista explicitado en el discurso de la política colombiana, corrobora las teorías sobre la extensión de la nación más allá de las fronteras explicadas anteriormente, y el énfasis en vincular Migración y Desarrollo por parte de los gobiernos de los Estados-nación emisores.

Y como consecuencia de la necesidad de buscar alianzas entre colombianos y colombianas, se fomenta también de forma explícita el asociacionismo de los migrantes en los países de destino. Tanto si se pertenece a la asociación como si no se pertenece, el migrante puede relacionarse con personas residentes en su mismo lugar de destino accediendo a la red creada por cada asociación en la plataforma RedEsColombia. "Hacer parte de RedEsColombia es contar con un canal para (...) vincularse con otros colombianos en su lugar de residencia" (Tour virtual, RedEsColombia). Este discurso está relacionado con el objetivo de fomentar la comunidad colombiana a lo largo de todo el mundo: cuanto mayor sea la relación de los emigrados entre sí, la vinculación con el país se retroalimentará de una forma natural, por contacto directo con las historias, intereses y proyectos de otros colombianos. El Portal ofrece la posibilidad de conocer personas que residen en el mismo lugar y ponerlas en contacto fácilmente.

Para $\mid$ González, Y. (junio, 2012). Migración transnacional colombiana y estrategias políticas online. 


\section{Conclusiones}

La utilización en los países de origen de las nuevas tecnologías de la información y la comunicación se ha convertido en una estrategia clara para la difusión y extensión de la nación más allá de las fronteras del Estado, que pretende propiciar un sentimiento de pertenencia y comunidad.

El estudio de las redes y usuarios del Portal RedEsColombia busca contestar a la pregunta de si los objetivos y estrategias desplegados por la política migratoria colombiana habían sido conseguidos a la luz de los datos empíricos que ofrece el desarrollo de RedEsColombia.

Como se ha visto, y en términos de lo que puede considerarse éxito o fracaso cuantitativo de la iniciativa política, queda claro que el incremento cuantitativo del número de redes y de usuarios registrados en el Portal no es directamente proporcional a la significatividad de dichas redes y a la dimensión de la actividad dentro de ellas. Y esto es porque la iniciativa pública es realmente el motor del Portal, en vez de ser las redes privadas creadas por migrantes. Además, los colombianos usuarios de esta plataforma no son personas emigradas sino nacionales que residen en el interior del país.

Por otro lado, el análisis cualitativo indaga sobre la explicación de las motivaciones de la política migratoria colombiana y de las consecuencias que ella tiene en la vida trasnacional de los migrantes, partiendo de la idea de que la política migratoria surge como reacción a una realidad que poco tiene que ver con lo migratorio, sino que busca implícitamente dar salida a las preocupaciones sobre política económica y de asuntos internos de los países de origen.

De ese análisis, se concluye que la migración colombiana vista desde los poderes del Estado sigue destilando una idea utilitarista; es decir, que la salida de personas del país constituye una oportunidad para el Desarrollo, así como una ocasión para la internacionalización y la mejora de la imagen de Colombia, y le otorga al migrante una responsabilidad individual y colectiva en esta materia. Sin embargo, en la actualidad, el Estado es también consciente de que es imprescindible para lograr estos objetivos, una gestión eficaz de esta migración a través de programas de retorno (físico o virtual) del capital humano, de la incentivación del asociacionismo migrante en destino y de la formación de comunidad más allá de las fronteras del Estado-nación. 


\section{Referencias}

Anderson, B. (1993). Comunidades imaginadas: Reflexiones sobre el origen y la difusión del nacionalismo. México: Fondo de Cultura Económica.

Beck, U. (2005). La mirada cosmopolita o La Guerra es la paz. Barcelona: Paidós.

Colombia Nos Une. Recuperado de http://www.minrelext.gov.co/wps/portal/colnosune

CONPES 3603. Consejo Nacional de Política Económica y Social, República de Colombia, Departamento Nacional de Planeación, Recuperado de http://www.cancilleria.gov.co/wps/wcm/connect/48ae9f004f72af348a20ea23813bba7f/DOCUMENTOCONPESPOLITICAMIGRATORIA.pdf?MOD=AJPERES\&CACHE ID=48ae9f004f72af348a20ea23813bba7f

Cortés, A., y Sanmartín, A. (2008). La dimensión transnacional: Teoría y práctica. En Fernández, M., Giménez, C. y Puerto, L. (Eds.), La construcción del codesarrollo (pp. 49-64). Madrid: La Catarata.

Departamento Nacional de Planeación y Sistema Naciones Unidas (2005). Hacia una Colombia equitativa e incluyente. Colombia Objetivos de Desarrollo del Milenio 2005, Bogotá.

Faist, T. (1999). Transnationalization in international migration: Implications for the study of citizenship and culture. University of Oxford: ISCA.

Garay, L., y Medina, M. (2008). La migración colombiana a España. El capitulo más reciente de una historia compartida. Madrid: OPI, Secretaría de Estado de Inmigración y Emigración, Ministerio de Trabajo e Inmigración.

Glick, N., Basch, L., \& Szanton Blanc, C. (1992). Towards a transnational perspective on migration: Race, class, ethnicity and nationalism reconsidered. Annals of the New York Academy of Sciences, 645, 1-24.

Guarnizo, L., Portes, A., \& Haller, W. (2003). Assimilation and transnationalism: Determinants of transnational political action among contemporary immigrants. American Journal of Sociology, 108(1), 1211-1248.

Portes, A. (2005). Convergencias teóricas y evidencias empíricas en el estudio del transnacionalismo de los inmigrantes. Migración y Desarrollo, 2-19.

Portes, A. (2009). Migration and development: Reconciling opposite views. Ethnic and Racial Studies, 32(1), 5-22.

Portes, A., Escobar, C., \& Walton Radford, A. (2007). Immigrant transnational organizations and development: A comparative study. International Migration Review, 41(1), 242-281. 
Portes, A., Haller, W., \& Guarnizo, L. (2002). Transnational entrepreneurs: An alternative form of immigrant adaptation. American Sociological Review, 67(2), 278-298.

Pries, L. (1999). La migración internacional en tiempos de globalización. Varios lugares a la vez. Nueva Sociedad, (164), 56-68.

Wimmer, A. y Glick Schiller, N. (2003). Methodological Nationalism, the Social Sciences and the Study of Migration: An Essay in Historical Epistemology. International Migration Review, 67, 576-610.

Para $\quad$ González, Y. (junio, 2012). Migración transnacional colombiana y estrategias políticas online. Reproducción de la comunidad a través de la vinculación transnacional. Ánfora, 19 (32), artículo: 69 - 90. Universidad Autónoma de Manizales. ISSN 0121-6538. 